\title{
Subject Index to Volume 35
}

Adolescent

bone mineral density, lumbar spine, age, sex, and puberty changes in, 362

prepubertal males with Tanner IV or V genital development, flutamide administration, 102

Adrenal steroidogenesis, abnormal, growthretarded newborns, 633

Adult respiratory distress syndrome, see Respiratory distress syndrome, adult

Amino acid

branched-chain, removal of, by peritoneal dialysis, as treatment for maple syrup urine disease, 357

cerebral transport, intrauterine growth retardation effects, developmental effects, 640

Amniotic fluid, IL-1 receptor antagonist concentration, fetal gender influence, 130

Androgen, role, altering gonadotropin secretion and elimination, pubertal male, 102

Anemia

of prematurity, erythropoietin production deficiency role as cause, 169

role, cause of hydrops, fetal sheep, 560

Angioedema, diagnosis of, in neonates, 184

Angiotensin II, role, development of hypertension, rat, 671

Anoxia

immature kidney resistance to, 152

ventilatory control, infant rat, 490

Antibody-dependent cellular cytotoxicity, fetal, to herpes simplex virus-infected cells, 289

Antiendotoxin monoclonal antibody, cytokine release and, blood leukocytes, 725

Antioxidant enzyme activity, increase in, in late gestation, 188

ARDS, see Respiratory distress syndrome, adult

Asphyxia, neonatal, magnetic resonance spectroscopy, lactate signal and $\mathrm{N}$-acetyl-aspartate/choline ratios, 148

Atrial natriuretic peptide, effects, vascular permeation, ovine fetus, 555

Behavior, early treatment for phenylketonuria effects on, 611

Bezold-Jarisch reflex, hemodynamic effects, veratridine effects, fetal lamb, 550

Bifidobacterium bifidum

effectiveness, rotavirus diarrhea, mouse, 690

intestinal colonization relationship with exogenous and endogenous growthpromoting factors, infants, 696

Blood flow

atrial myocardial, during right ventricular pressure load and adenosine infusion, fetal sheep, 325

brain, and carotid arterial flow, fetal sheep, 329

breathing movements effects on, fetal sheep, 484

cerebral

prostaglandins and endothelium-derived relaxation factor role in regulation. piglet, 649 responses to indomethacin, newborn pig, 565

velocity, cerebral, fluctuations in, and sleep state relationship, newborn, 50

Body, composition, bioelectric impedance analysis, fat-free mass estimating, 617

Bone

demineralization, postnatal, prevention of, VLBW infant, with calcium and phosphorus supplementation, 125

mineral density

deficiency of, role, causing dolichocephalic head flattening, VLBW infants, 701

lumbar spine, age, sex, and puberty changes, children and adolescents, 362

Brain, magnetic resonance spectroscoy, L-2 hydroxyglutaric acidemia patient, 614

Breathing movements, fetal, effects on blood flow, fetal sheep, 484

Bronchopulmonary dysplasia, ventilatory responses to hypoxia in infants with. SIDS implications, 677

Calcium

antagonist, therapy, role, treatment for pre natal hypoxia-ischemia, fetal sheep, 657

supplementation, role, preventing postnatal bone demineralization, VLBW infant, 125

L-Carnitine, intravenous, role, enhancing of amino acid oxidation, patients with inborn errors of metabolism, 96

Central nervous system, vascular lesions of, pathogenesis of, VLBW infant, 424

Cerebral artery, sensitivity of, enhanced by maturation, to potassium channel activator lemakalim, 729

Children

bone mineral density, lumbar spine, age, sex, and puberty changes in, 362

cystic fibrosis, energy expenditure and genotype, 451

growth-retarded, procollagen rhythms in, 409

hospitalized for psychiatric disease, triglyceride and total plasma cholesterol relationships with, 602

leukemia, insulin-like growth factor bind ing protein-3, concentrations and protease activity, 720

prepubertal, growth after renal transplantation, 367

sepsis, phenylalane and tyrosine kinetics in, 580

Cholesterol esters, in umbilical cord blood, essential fatty acid gestational age dependency, 461

Cholesterol, synthesis rates, infant nutrition effects on, 135

Cirrhosis, Indian childhood, metallothionein synthesis and degradation in, 197

Cocaine, prenatal exposure to, cerebral responses, newborn sheep, 339

Colon, smooth muscle, response to electrical field stimulation, newborn and adult rabbits, 470

Complement C9, supplement, role, enhanc735 ing ability neonatal serum in killing Escherichia coli, 389

Copper, homeostasis, after onset of hepatitis rat, 598

Corticosteroids, effects, lung maturation, preterm lamb, 479

Crigler-Najjar syndrome type I, bilirubin uridine 5 '-diphosphate-glucuronosyltransferase gene defect, 629

Cystic fibrosis

energy expenditure and genotype, children, 451

transmembrane conductance regulator, developmental differences, airway epithelial cells, fetus, rat, 45

Cystine, intracellular distribution of, proximal tubules, 447

Cytochrome $\mathrm{P} 450$, imprinting, in adulthood, neonatal hyperoxia effects on, 255

Cytokines, release, blood leukocytes, 725

Development, intrauterine growth retardation effects, cerebral amino acid transport, 640

Dexamethasone

cytokine release and, blood leukocytes, 725

prenatal exposure to, role, causing loss of hypoxia tolerance, neonate, 515

Diabetes mellitus, effects, on maternofetal flux of calcium and magnesium, rat placenta, 376

Diabetes, influence of, on epidermal growth factor in milk, 107

Dialysis, role, removal of branched-chain amino acids, as treatment for maple syrup urine disease, 357

Diarrhea, rotavirus, Bifidobacterium bifidum effectiveness in, mouse, 690

Dopamine receptors, functional ontogeny of, perfusion lung, rabbit, 228

Echocardiography, Doppler, role, evaluating ventricular output and diastolic filling changes, neonate, 506

Electrical field stimulation, colonic smooth muscle response to, newborn and adult rabbits, 470

Endothelin, role, regulation fetal pulmonary vascular tone, 664

Endothelium-derived relaxation factor, role, regulation of cerebral blood flow and oxygen utilization, piglet, 649

Endotoxemia, gut-derived, modification by ursodeoxyholic acid, neonatal rat, 214

Endotoxin, Escherichia coli, role, depressing ventricular contractility, neonatal lambs, 62

Energy, expenditure, children with cystic fibrosis, 451

Enzyme, imprinting, in adulthood, neonatal hyperoxia effects, rat, 255

Epidermal growth factor

decreased level, in milk from diabetic rats, 107

prenatal exposure, role, attenuating respiratory distress syndrome, premature monkey, 30

Epithelial cells, airway, developmental differences of cystic fibrosis transmembrane conductance regulator, fetal rat, 45

Erythropoietin, deficiency, role, cause of ane- 
mia of prematurity, preterm infants, 169

Escherichia coli

complement component $\mathrm{C}$, role, enhancing ability neonatal serum in killing, 389

endotoxin, role, depressing ventricular contractility, neonatal lambs, 62

Exercise, training, growth hormone suppression effects, rat, 223

Fat-free mass, bioelectric impedance analysis of, in children, 617

Fetal hematopoietic progenitors, human, stem cell factor actions on, 303

$\alpha$-Fetoprotein, levels, role, detection of, he-

Fetus reditary tyrosinemia type I, 205

atrial natriuretic peptide effects on vascular permeation, sheep, 555

Bezold-Jarisch reflex, veratridine effects on hemodynamics, lamb, 550

breathing movements effects on blood flow, sheep, 484

cystic fibrosis, transmembrane conductance regulation, developmental differences, rat, 45

gender influence, IL-1 receptor antagonist concentration, in amniotic fluid and newborn urine, 130

heart rate fluctuations, mechanisms regulating, 250

hemolysis, in alloimmunized pregnancy, in alloimmunized pregnancy, 713

hydrops in, role of anemia, sheep, 560

hypoxia-ischemia, calcium antagonist therapy role in treating, sheep, 657

iopanoic acid effects on 5 '-deiodinase, fetal tissues, rat, 91

kidney, manganese superoxide dismutase expression, during development, sheep, 41

pulmonary vascular tone, endothelin role in regulating, sheep, 664

Fibroblasts

cirrhosis, metallothionein synthesis and degradation, 197

skin, glucose and galactose toxicity effects on transport and metabolism in, 141

Flunarizine, role, treating prenatal hypoxiaischemia, fetal sheep, 657

Fluoride, pharmacokinetics, in infants, 157

Flutamide, administration, prepubertal males with Tanner IV or $\mathrm{V}$ genital development, 102

Functional residual capacity, measurement of, during mechanical ventilation and spontaneous breathing, small-volume lungs, 494

Galactose, toxicity, effects on transport and metabolism, skin fibroblasts, 141

Glucocorticoids

maturational effects of, proximal tubules, 474

role, correcting pulmonary immaturity, congenital diaphragmatic hernia, 523

Glucose

absorption, intestinal, cystic fibrosis, loperamide inhibition of, 354

toxicity, effects on transport and metabolism, skin fibroblasts, 141

Glutathione

peroxidase, expression, organs, mouse embryos, fetuses, and neonates, 188

reductase, endotoxin induction of, lungs, mice, 311

synthetase, deficiency, impaired lipoxygenase products synthesis, 307
Glycogen storage disease, granulocyte colony-stimulating factor, effects on neutrophils, 84

Granulocyte colony-stimulating factor, effects on neutrophils, glycogen storage disease type 1B, 84

Granulocyte-macrophage colony-stimulating factors, in cord and maternal serum, at delivery, 164

Growth

after renal transplantation, prepubertal children, 367

response, growth hormone suppression effects, rat, 223

retardation, intrauterine

abnormal adrenal development and function, newborns, 633

developmental effects, on cerebral amino acid transport, 640

effects on heart rate and heart rate variability, 500

-promoting factors, exogenous and endogenous, Bifidobacterium bifidum intestinal colonization relationship with, infants, 696

Growth factor, nerve, levels of, umbilical cord blood, newborns, 637

Growth hormone

bioactivity, girls with Turner's syndrome, insulin-like growth factor I correlation, 218

deficiency, procollagen rhythms in, children, 409

suppression, effects, exercise training and growth response, rat, 223

Haemophilus influenzae type B, leukocytes stimulated with, cytokine release, 725

Head flattening, dolichocephalic, bone mineral deficiency as cause of, VLBW infants, 701

Heart

disease, congenital, heparin therapy, pediatric patients, evaluation of efficacy and safety, 78

rate, and heart rate variability, during sleep, small-for-gestational age infants, 500

rate, fluctuations, mechanisms regulating, fetal sheep, 250

vasoactive intestinal peptide, electrophysiologic activity, newborn, 244

Hemodynamics, intracranial, during and after hyperventilation, monitoring with near-infrared spectroscopy, newborn rabbit, 334

Hemolysis, carboxyhemoglobin relation with, in alloimmunized pregnancy, 713

Heparin therapy, pediatric patients, evaluation of efficacy and safety, 78

Hepatitis, copper homeostasis after onset of, rat, 598

Hernia, congenital diaphragmatic, glucocorticoid role, correcting pulmonary immaturity, 523

Herpes simplex virus, antibody-dependent cellular cytotoxicity, human fetus, 289

Homocysteine, export, effect of methionine and nitrous oxide, cystathionine synthase-deficient patients, 1

Hyaluronan deposition, changes, during respiratory distress syndrome, premature monkeys, 238

Hydrophobicity

skin surface, isopropanol effects on, newborn, 443

surface, of rabbit stomach, 209
Hydrops, anemia role as cause of, fetal sheep, 560

Hyperbilirubinemia, Crigler-Najjar syndrome type I patients, 629

Hyperoxia

lung damage after, propylthiouracil treatment, 530

neonatal, effects, cytochrome P450 imprinting in adulthood, 255

preterm versus full-term newborn responses, rat, 233

Hypertension

hypoxic pulmonary nitric oxide role reversing, newborn pig, 15

renin-angiotensin system role in development of, rat, 671

Hyperventilation, intracranial hemodynamics and oxygenation during and after, monitoring with near-infrared spectroscopy, newborn rabbit, 334

Hypophosphatemia, X-linked, chromosomal localization renal sodium phosphate transporter, implications, 510

Hypothermia, role, providing neuroprotection for ischemia, neonatal brain, 436

Hypoxia

neonatal, loss of tolerance to, role, prenatal exposure to dexamethasone, 515

vascular site of action, neonatal lung, pig, 25

ventilation and oxygen consumption during, newborn pig, 536

ventilatory response to, amino acid effects on, neonatal pig, 316

Hypoxia-ischemia

inhibitor of nitric oxide, role, reducing, neonatal rat, 10

prenatal, calcium antagonist therapy, fetal sheep, 657

Ig, see Immunoglobulin

Immunoglobulin, intravenous, role, modulating production of TNF- $\alpha, 397$

Indomethacin, cerebral blood flow responses to, newborn pig, 565

Infant, see also Neonate

angioedema, hereditary, diagnosis, 184

anoxia, ventilatory control after, rat, 490

fluoride pharmacokinetics, 157

hyperoxia, responses to, versus preterm infant, rat, 233

lactoferrin influence on iron absorption from human milk, 117

low birth weight, protein and energy intakes, mathematical model for predicting weight gain composition, 704

postnatal lung growth, alveolar surface area, surfactant protein $A$, and phosphatidylcholine changes, rat, 685

prenatally exposed to cocaine, cerebral responses in newborn, sheep, 339

preterm

anemia of prematurity, erythropoietin production deficiency role as cause, 169

corticosteroids effects, lung maturation, lamb, 479

hyperoxia, responses to, versus full-term newborn, rat, 233

RDS, surfactant treatment for, changes in lung mechanics during, 541

respiratory distress syndrome, hyaluronan deposition changes during, 238

respiratory distress syndrome, prenatal exposure to epidermal growth factor role attenuating, premature monkey, 30

small-for-gestational age, heart rate and heart rate variability during sleep, 500 


\section{SUBJECT INDEX TO VOLUME 35}

ventilatory responses to hypoxia in infants with bronchopulmonary dysplasia. SIDS implications, 677

very low birth weight

bone mineral deficiency, role, causing dolichocephalic head flattening, 701

histochemical staining, role, studying germinal matrix hemorrhage, and brain vascular morphology, 424

nerve growth factor levels, umbilical cord blood, 637

postnatal bone demineralization prevention, with calcium and phosphorus supplementation, 125

Insulin-like growth factor, binding protein-3, concentrations and protease activity, malignant solid tumor and leukemia patients, 720

Insulin-like growth factor I, correlation, with growth hormone bioactivity, girls with Turner's syndrome, 218

Insulin-like growth factor II, zinc suppression role in decreasing, 404

Intrauterine growth retardation, see Growth

Iopanoic acid, effects on 5 '-deiodinase, fetal tissues, rat, 9

Iron, absorption, lactoferrin influence on, from human milk, infants, 117

Ischemia, role, hypothermia providing neuroprotection, neonatal brain, 436

IUGR, see Growth, retardation, intrauterine

Kawasaki disease, role, heat shock protein in, polymerase chain reaction determination, 179

Kidney

immature, resistance to anoxia, 152

manganese superoxide dismutase expression, during development, sheep, 41

transplantation, growth after, prepubertal children, 367

$\alpha$-Lactalbumin, human, and bovine $\beta$-lactoglobulin absorption, in premature infants, 344

$\beta$-Lactams, Pseudomonas cepacia susceptibility to, $\mathrm{pH}$ and $\mathrm{CO}_{2}$ effects on, children with cystic fibrosis, 293

$\beta$-Lactoglobulin, bovine, and human $\alpha$-lactalbumin absorption, in premature infants, 344

Lactoferrin, influence on iron absorption from human milk, infants, 117

Legg-Perthes disease, protein $\mathrm{C}$ and $\mathrm{S}$ deficiency, thrombophilia, and hypofibrinolysis, as pathophysiologic causes of, 383

Leukemia

childhood, CNS therapy for, steroid, methotrexate and radiation neurotoxicity interactions, 171

insulin-like growth factor binding protein3 , concentrations and protease activity, children with, 720

Leukocytes, blood, cytokine release by, 725

Lipoxygenase, synthesis, impaired, in glutathione synthetase deficiency, 307

Loperamide, inhibiting, intestinal glucose absorption, cystic fibrosis, 354

Lung

damage, after hyperoxia, propylthiouracil treatment for, 530

glutathione reductase activity, endotoxin induction of, mice, 311

growth, postnatal, alveolar surface area, surfactant protein $A$, and phosphatidylcholine changes, rat, 685

malic enzyme activity in, adult and newborn rat, 589 maturation, corticosteroids role in altering, preterm lambs, 479

perfused, ontogeny of dopamine receptors, rabbit, 228

vascular site of action of hypoxia, neonatal pig, 25

Magnetic resonance spectroscopy

brain, L-2-hydroxyglutaric acidemia patient, 614

${ }^{31} \mathrm{P}$, phosphorus metabolite concentrations, human brain, 431

Malic enzyme, activity, in lung, adult and newborn rat, 589

Maple syrup urine disease, treatment for, 357

Maternal serum, granulocyte-macrophage colony-stimulating factors in, at delivery, 164

Metabolism

inborn errors of, intravenous L-carnitine, role, enhancing of amino acid oxidation, 96

lipoprotein, dietary nucleotides influence on, newborns, 112

Metallothionein, synthesis and degradation, Indian childhood cirrhosis, 197

Methionine synthase, effect, homocysteine export, cystathionine synthase-deficient patients, 1

Methotrexate, effects, altering radiation effects on growth, rat, 416

Milk, human, lactoferrin influence on iron absorption, infant, 117

Neonate, see also Infant

angioedema, hereditary, diagnosis, 184

asphyxiated, lactate signal and $N$-acetylaspartate/choline ratios, using magnetic resonance spectroscopy, 148

cerebral blood flow response to indomethacin, pig, 565

decreased tolerance to hypoxia, role, prenatal exposure to dexamethasone, 515

dietary nucleotides influence on lipoprotein metabolism, newborns, 112

Doppler echocardiographic evaluation of, ventricular output and diastolic filling changes, 506

endotoxemia, ursodeoxycholic acid role in modifying, rat, 214

heart, electrophysiologic activity, vasoactive intestinal peptide, 244

hyperoxia, effects, cytochrome P450 im-

hypoxia

vascular site of action, neonatal lung, pig, 25

ventilatory response to, amino acid effects on, pig, 316

hypoxia-ischemia, inhibitor of nitric oxide, role, reducing, neonatal rat, 10

inhaled nitric oxide effects, pulmonary circulation, pig, 20

ischemic, hypothermia role, providing neuroprotection for, brain, 436

lung, malic enzyme activity in, rat, 589

oxygen consumption and ventilation changes in, during hypoxia, pig, 536

rotavirus diarrhea, Bifidobacterium bifidum effectiveness in, mouse, 690

sepsis, GM-CSF and G-CSF role in management of, 164

skin surface hydrophobicity, isopropanol effects on, 443

sleep state and cerebral blood flow velocity fluctuations relationship, 50

Neurotoxicity, associated with CNS therapy, for childhood leukemia, 171 printing in adulthood, 255
Nitric oxide

effects on homocysteine export and remethylation, cystathionine synthasedeficient patients, 1

inhaled, effects, pulmonary circulation, neonatal pig, 20

role, reversing hypoxic pulmonary hypertension, newborn pig, 15

synthesis, inhibitor of, role, reducing hypoxic-ischemic brain damage, neonatal rat, 10

Nucleotides, dietary, role, influencing lipoprotein metabolism, newborns, 112

Nutrition, effects on cholesterol synthesis rates, infant, 135

Obesity, defective stimulation of adipocyte adenylate cyclase, role causing, in pseudohypoparathyroidism la

Oxygen

consumption, changes in, during hypoxia, newborn pig, 536

inspired, respiratory response to alterations in, newborns, 321

utilization, endothelium-derived relaxation factor and prostaglandins rule in regulating, 649

Oxygenation

during and after hyperventilation, monitoring with near-infrared spectroscopy, newborn rabbit, 334

hypoxemia, with ARDS, peritoneal ventilation treatment, 682

Pentoxifylline, cytokine release and, blood leukocytes, 725

Pharmacokinetics, fluoride, in infants, 157

Phenylalane, kinetics, children with sepsis, 580

Phenylketonuria, personality characteristics, patients treated early, 611

Phosphorus, supplementation, role, preventing postnatal bone demineralization. VLBW infant, 125

Placenta, diabetes mellitus effects, maternofetal flux of calcium and magnesium, rat, 376

Polymerase chain reaction, role, determining heat shock protein expression, Kawasaki disease, 179

Prednisolone, effects, altering radiation effects on growth, rat, 416

Pregnancy, alloimmunized, fetal carboxyhemoglobin with hemolysis relation with, 713

Propylthiouracil, treatment, lung damage after hyperoxia, 530

Prostaglandins, role, regulation of cerebral blood flow and oxygen utilization, piglet, 649

Protein C, deficiency, role, as cause of LeggPerthes disease, 383

Protein S, deficiency, role, as cause of LeggPerthes disease, 383

Protein, heat shock, role, Kawasaki disease, polymerase chain reaction determination, 179

Proximal tubules

increase in, glucocorticoids role in, 474

intracellular distribution of cystine, 447

Pseudohypoaldosteronism, reduced $\mathrm{Na}^{+}, \mathrm{K}^{+}$. ATPase activity in patients with, 372

Pseudohypoparathyroidism la, defective stimulation of adipocyte adenylate cyclase, role, decreased lipolysis and obesity, 594

Pseudomonas aeruginosa, $\mathrm{IgG}$ and complement contributions to phagocytosis of, 68 
Pseudomonas cepacia, susceptibility to $\beta$-lactams, $\mathrm{pH}$ and $\mathrm{CO}_{2}$ effects on, children with cystic fibrosis, 293

Psychiatric disease, suicide and suicide ideation, children hospitalized for, total plasma cholesterol and triglyceride relationships, 602

Psychosocial consequences, phenylketonuria, early treatment effects on, 611

Pulmonary circulation, inhaled nitric oxide effects, neonatal pig, 20

Pulmonary embolism, heparin therapy, pediatric patients, evaluation of efficacy and safety, 78

Pulmonary vascular tone, endothelin role in regulating, fetal sheep, 664

Radiation, effects on growth, prednisolone and methotrexate effects on, rat, 416

RDS, see Respiratory Distress Syndrome

Respiratory distress syndrome

adult, peritoneal ventilation treatment, 682

hyaluronan deposition changes, preterm monkey, 238

prenatal exposure to epidermal growth factor role attenuating, premature monkey, 30

surfactant treatment, changes in lung mechanics during, 541

Rickets, vitamin D-resistant, chromosomal localization renal sodium phosphate transporter, implications, 510

Salivary glands, increased epithelial expression of HLA-DR, role in SIDS, 625

Sepsis

neonatal, GM-CSF and G-CSF role in management of, 164

phenylalane and tyrosine kinetics in, children with, 580

SIDS, see Sudden infant death syndrome

Sinus node, response maturation, to vagal stimulation, dog, 55

Skin surface, hydrophobicity, isopropanol ef-

Sleep fects on, newborn, 443

heart rate and heart rate variability, smallfor-gestational age infants, 500

state, and cerebral blood flow velocity fluctuations relationship, newborns, 50

\section{Spectroscopy}

near-infrared, role, monitoring intracranial hemodynamics and oxygenation, during and after hyperventilation, newborn rabbit, 334

proton magnetic resonance

brain, L-2-hydroxyglutaric acidemia patient, 614

lactate signal and $N$-acetyl-aspartate/ choline ratios, asphyxiated full-term neonate, 148

${ }^{31} \mathrm{P}$ magnetic resonance, phosphorus me- tabolite concentrations, human brain, 431

Spine, lumbar, bone mineral density changes in, age, sex, and puberty related, children and adolescents, 362

Staphylococcal, enterotoxin B, proliferative and cytokine response, $\mathrm{T}$ cells, stimulated with, newborn, 293

Stem cell factor, actions on human fetal hematopoietic progenitors, 303

Stomach, surface hydrophobicity properties of, 209

Streptococcus, group B, infection, thromboxane role in mediating pulmonary response to, 571

Sudden infant death syndrome

increased epithelial expression of HLA-DR role in, 625

ventilatory responses to hypoxia in infants with bronchopulmonary dysplasia, implications, 677

Suicide, children, hospitalized for psychiatric disease, triglyceride and total plasma cholesterol relationships with, 602

Superoxide dismutase

copper/zinc

and exogenous lung surfactant, pharmacologic interactions, 37

expression, organs, mouse embryos, fetuses, and neonates, 188

manganese, expression in kidney cortex during development, sheep, 41

Surfactant

lung, and copper/zinc superoxide dismutase, pharmacologic interactions, 37

protein $\mathrm{A}$, changes in, with postnatal lung growth, rat, 685

therapy, RDS, changes in lung mechanics during, 541

T cells, proliferative and cytokine response, stimulated staphylococcal enterotoxin B, newborn, 293

TGF- $\alpha$, see Transforming Growth Factor- $\alpha$

Thrombophilia, role, as cause of LeggPerthes disease, 383

Thrombosis, deep venous, heparin therapy, pediatric patients, evaluation of efficacy and safety, 78

Thromboxane, role, mediating pulmonary response to group B streptococcus infection, 571

TNF- $\alpha$, see Tumor necrosis factor- $\alpha$

Transforming growth factor- $\alpha$, presence in small intestine and pancreas, absence in milk, rat, 348

Transplantation, kidney, growth after, prepubertal children, 367

Triglycerides

in umbilical cord blood, essential fatty acid gestational age dependency, 461

relation to suicide, suicide ideation and hospitalization for psychiatric disease, children, 602

Tumor necrosis factor- $\alpha$, production, intravenous immunoglobulin role in modulating, 397

Tumors, malignant solid, children, insulinlike growth factor binding protein-3, concentrations and protease activity, 720

Turner's syndrome, growth hormone bioactivity, girls with, insulin-like growth factor I correlation, 218

Tyrosinemia type 1 , hereditary, $\alpha$-fetoprotein serum levels, role, detection of, 205

Tyrosine, kinetics, children with sepsis, 580

Umbilical cord blood

cholesterol esters and triglycerides, essential fatty acid gestational age dependency, 461

granulocyte-macrophage colony-stimulating factors in, at delivery, 164

nerve growth factor levels, newborns, 637

Urine, IL-1 receptor antagonist concentration, fetal gender influence, 130

Ursodeoxycholic acid, role, modifying endotoxemia, neonatal rat, 214

Vagal stimulation, sinus node response maturation, dog, 55

Vascular permeation, atrial natriuretic peptide effects on, ovine fetus, 555

Vascular reactivity, maturational changes in, arterial electrophysiologic characteristics, 729

Vasoactive intestinal peptide, electrophysiologic activity, newborn heart, 244

Ventilation

changes in, during hypoxia, newborn pig, 536

mechanical, functional residual capacity measurement, small-volume lungs, 494

peritoneal, treatment for ARDS, 682

Ventilatory control, after anoxia, infant rat, 490

Ventricular contractility, Escherichia coli endotoxin role, depressing, neonatal lambs, 62

Ventricular output, Doppler echocardiographic evaluation of, neonate, 506

Ventricular pressure, load, and adenosine infusion, atrial myocardial blood flow, fetal sheep, 325

Veratridine, effects on hemodynamics, Bezold-Jarisch reflex, fetal lamb, 550

VLBW, see Infant, very low birth weight

Weight gain, low birth weight infant, proteins and energy intakes, mathematical model for predicting composition of, 704

Wilson's disease, hepatic, reduced biliary excretion of copper, 598

Zinc, suppression, role, decreasing insulinlike growth factor II, 404 